Canadian University Music Review

Canadian University Music Review

Revue de musique des universités canadiennes

\title{
Les femmes musiciennes sous les Bourbon d'après les documents inédits de Marie Bobillier
}

\section{Albert La France}

Volume 16, numéro 1, 1995

Voices of Women: Essays in Honour of Violet Archer

Voix de femmes : mélanges offerts à Violet Archer

URI : https://id.erudit.org/iderudit/1014416ar

DOI : https://doi.org/10.7202/1014416ar

Aller au sommaire du numéro

\section{Éditeur(s)}

Canadian University Music Society / Société de musique des universités canadiennes

\section{ISSN}

0710-0353 (imprimé)

2291-2436 (numérique)

Découvrir la revue

Citer cet article

La France, A. (1995). Les femmes musiciennes sous les Bourbon d'après les documents inédits de Marie Bobillier. Canadian University Music Review / Revue de musique des universités canadiennes, 16(1), 60-73.

https://doi.org/10.7202/1014416ar
Résumé de l'article

À sa mort en 1918, Marie Bobillier (pseud. Michel Brenet) a laissé au Département des manuscrits de la Bibliothèque nationale à Paris un nombre impressionnant de notes, de citations et de relevés qu'elle avait accumulés au cours de sa carrière et qui, pour la plupart, demeurent inédits. Dans un dépouillement de ces volumes, l'auteur a extrait les citations, les rapports et les commentaires des auteurs des trois derniers siècles, tous sous le thème « femmes musiciennes ». Il en résulte un éventail assez étonnant des activités musicales des femmes de l'ancien régime sous les rois Bourbon, une espèce de sondage du passé à partir duquel tirer quelques conclusions plus éclairées. Malheureusement, le travail n'a servi qu'à confirmer ce que d'autres ont déjà conclu, à savoir que les femmes des $\mathrm{XVII}^{\mathrm{e}}$ et XVIII ${ }^{\mathrm{e}}$ siècles n'avaient qu'un rôle secondaire en musique, tout comme dans les autres aspects de la société, soit artistique, politique ou social. Il faudra attendre le $\mathrm{XX}^{\mathrm{e}}$ siècle pour que les femmes puissent commencer à prendre leur juste place dans les rangs de la profession musicale.
All Rights Reserved @ Canadian University Music Society / Société de musique des universités canadiennes, 1995
Ce document est protégé par la loi sur le droit d'auteur. L'utilisation des services d'Érudit (y compris la reproduction) est assujettie à sa politique d'utilisation que vous pouvez consulter en ligne.

https://apropos.erudit.org/fr/usagers/politique-dutilisation/ 


\section{LES FEMMES MUSICIENNES SOUS LES BOURBON D'APRÈS LES DOCUMENTS INÉDITS DE MARIE BOBILLIER}

\section{Albert La France}

Michel Brenet, nom bien connu du monde de la musique française, est le pseudonyme dont s'est servi Marie Bobillier (1858-1918) dans ses nombreux écrits et publications. L'une des premières femmes musicologues françaises, son œuvre porte sur divers aspects historiques de la musique, à partir du Moyen Âge jusqu'au XIX' ${ }^{\mathrm{e}}$ siècle. À sa mort, en 1918, elle a laissé au Département des manuscrits de la Bibliothèque nationale à Paris un nombre impressionnant de notes, de citations et de relevés qu'elle avait accumulés au cours de sa carrière et qui, pour la grande part, demeurent inédits. Ces ouvrages, reliées en 19 tomes, portent le nom collectif de Documents sur la musique ${ }^{1}$. Un parcours rapide de ces volumes révèle la récurrence du thème des "femmes musiciennes ». En effet, cette récurrence a été confirmée par le dépouillement systématique de ces documents dont l'analyse témoigne d'une gamme riche et variée d'activités musicales des femmes françaises sous l'ancien régime. Le résultat de ce dépouillement fait l'objet du présent article.

Des études touchant la situation des femmes musiciennes à cette époque ont déjà été entreprises par Marcelle Benoit et Julie Anne Sadie², et nous n'avons nullement l'intention de revenir là-dessus. Notre travail constitue plutôt un complément à ces études. Il présentera un éventail des activités musicales et des faits retenus par un certain nombre d'auteurs des trois derniers siècles et recueillis par Marie Bobillier.

Au début du règne des Bourbon, l'activité musicale des femmes de l'ancien régime se retrouve principalement chez les aristocrates dans un cadre amateur. Les femmes de la noblesse enclines à la musique, y compris les reines, les favorites des rois et les princesses, chantaient, jouaient du luth ou du clavecin, souvent pour leur propre plaisir et divertissement. L'établissement de l'Académie d'opéra, par Perrin en 1669, et repris sous le nom d'Académie royale de musique par Lully en 1772, permettra, avec la permission du Roi, aux Dames et aux Demoiselles de la noblesse et de la bourgeoisie de faire carrière profes-

1 Nouvelles acquisitions françaises, $\mathrm{n}^{\text {os }} 11407-11425$. Tout au cours de ces notes, nous renverrons à ces tomes par les lettres NAF entre crochets.

2Marcelle Benoit, Versailles et les musiciens du Roi, 1661-1733 : étude institutionnelle et sociale (Paris : Éditions A. et J. Picard, 1971) ; et Julie Anne Sadie, « Musiciennes of the Ancien Régime », dans Women Making Music: The Western Art Tradition, 1150-1950, édité par Jane Bowers et Judith Tick (Urbana et Chicago : University of Illinois Press, 1986) : 191-223. 
sionnelle comme chanteuses. Plus tard, les meilleures d'entre elles feront leur chemin à la cours, dans la Musique de la Chambre et éventuellement dans la Musique de la Chapelle du roi, dans les concerts publiques, notamment au Concert spirituel, fondé par Philidor en 1725. Le cheminement des femmes instrumentistes et des compositrices ne se fera que lentement et progressivement au cours du XVIII ${ }^{\mathrm{e}}$ siècle. Seules celles qui démontreront un talent extraordinaire pour leur instrument ou pour la composition jouiront éventuellement d'une certaine renommée ${ }^{3}$.

\section{Les musiciennes issues de la royauté et de la noblesse}

Chez les Bourbon, la musique avait, sans contredit, une importance majeure dans la formation des princes et des princesses. On choisit pour leur enseigner, les meilleurs maîtres du royaume, et certaines princesses se montreront plus douées que d'autres. Un cas en particulier est celui d'Henriette-Marie de France, fille d'Henri IV, qui épousa Charles Ir , roi d'Angleterre (1625-49). Elle avait, paraitt-il, le talent d'une musicienne de profession, comme en font foi les citations qui suivent :

Elle chante comme un ange, et le clavessin [sic] n'est jamais mieux touché que par ses belles mains $[\ldots]^{4}$

Cette princesse tenait de sa mère le goât des arts ; elle aimait les tableaux, la musique, l'architecture, le théâtre, les ballets, tout ce qui était beau et brillant. Douée d'une voix merveilleuse dont la douceur et l'étendue auraient pu faire d'elle, si elle n'eât été reine, la prima donna de l'Europe, elle se plaisait à la cultiver, et les meilleurs moments pour elle étaient ceux où elle pouvait se livrer sans contrainte à ses goûts favoris 5 .

On m' avait dit, et j'ai reconnu que c'était la vérité même, que ni son maître de chant, ni personne, homme ou femme en France, ou en Europe, ne chante aussi admirablement qu'elle ; sa voix dépasse toute imagination ; je ne saurais vous en dire d'avantage $^{6}$.

L'étiquette de la cour d'Angleterre interdisait à la reine de chanter en public ; elle chantait souvent dans ses appartements privés et parfois en berçant ses enfants ; c'était à qui, parmi les courtisans, serait assez heureux pour saisir au vol quelques notes?

3 Benoit, Versailles et les musiciens du roi, 254-60 ; Sadie, « Musiciennes of the Ancien Régime ", 191-97. Voir aussi Michel Brenet, Les concerts en France sous l'ancien régime (Paris : Librairie Fischbacher, 1900 ; réimpression, New York : Da Capo Press, 1970), 160-64.

4 « Portrait de la princesse d'Angleterre sous le nom de la princesse Cléopâte [sic], par Mme la comtesse de Bregy, juin 1658 ", Mémoires pour servir à l'histoire d'Anne d'Autriche, épouse de Louis XIII, roi de France par Madame de Motteville, une de ses favorites, 5 vol. (Amsterdam : François Chanquion, 1823), 5:114 [NAF 11408, 718]. Les citations sont données textuellement, telles qu'elles apparaissent dans les documents de Marie Bobillier.

5 Henriette-Marie de France, reine d'Angleterre, étude historique par le conte de Baillon, suivie de lettres inédites (Paris : Didier, 1877), 10 [NAF 11408, 870].

6Lettre de Lord Kensington au prince de Galles, plus tard Charles $I^{\text {ier }}$, sur Henriette-Marie..., 32 [NAF 11408, 780].

7 Agnes Strickland, Queens of England, 8 vol. (Londres : Colburn, 1851-54), 4:140 [NAF 11408, 870]. 
Au temps de Louis XIV, la dauphine manifestait un goût et un certain talent pour la musique, si l'on en croit les commentaires élogieux du Mercure galant :

[...] l'illustre M. Lambert. Monsieur l'a choisy pour apprendre à chanter à Mademoiselle. Il fallait ce grand homme pour perfectionner la belle voix de cette jeune princesse et pour mettre le comble de ses rares et extraordinaires qualitez [...] Elle danse avec un air charmant, touche le clavessin en mesure, et possede assez la Musique pour chanter à livre ouvert. On ne devait pas moins attendre des hommes choisis qui ont travaillé à son éducation. $\mathrm{M}^{\mathrm{r}}$ des Ais [du Désert] ${ }^{\mathrm{MB}}$ pour la danse ; feu $\mathrm{M}^{\mathrm{r}}$ Hardel et $\mathbf{M}^{\mathrm{r}}$ d'Anquelberg [Anglebert] ${ }^{\mathrm{MB}}$ pour le clavessin, et $\mathbf{M}^{\mathrm{r}}$ Clement pour la musique, sont ceux qui ont esté employez à luy montrer ces beaux arts ${ }^{8}$.

[La dauphine] chante agréablement et avec méthode, joue du clavessin, sçait un peu le Dessein, danse d'une manière aisée, et juge très bien de tout ${ }^{9}$.

Rien n'est si à la mode que la musique, et c'est aujourd'hui la passion de la pluspart des honnestes gens et des personnes de qualités ; mais peu y réussissent comme Madame la Dauphine. Cette princesse apprend à chanter depuis 5 ou 6 mois avec un succès surprenant. Elle a la voix d'une grande douceur, très légère, fort étendue, et le gozier du monde le plus flexible. Elle sçait parfaitement ce qu'elle chante, et c'est toujours avec le même esprit et la même grâce qui accompagnent tout ce qu'elle fait [...] Mad. la Dauphine a l'oreille admirable, et naturellement sûre, en sorte qu'elle chante parfaitement en partie, à deux, à trois, et avec accompagnement ; tout lui est égal, airs, scènes d'opéra, airs de Lambert, du Camus, du vieux Boësset et autres avec les doubles $[\ldots]^{10}$.

Parmi les personnes les plus illustres de la noblesse qui pratiquaient la musique on doit compter $\mathrm{M}^{\mathrm{me}}$ Marie de Hautefort (1616-91), fille d'honneur d'Anne d'Autriche, et favorite de Louis XIII.

Olympe [ $\mathrm{M}^{\mathrm{me}}$ de Hautefort $]$ a le ton et l'accent tendre et passionné [...] ; elle sait jouer de la guitare, touche l'angélique d'une manière extraordinaire; et si elle n'y avait rien négligé, on peut dire qu'elle y aurait excellé. Elle chante bien, et quoique sa voix ne soit pas des plus grandes ni des plus belles, l'oreille se tromperait assurément si on ne jugeait pas qu'elle est des plus douces et des plus charmantes $[\ldots]^{11}$

Mais, hélas, elle fut remplacée par une autre favorite qui, elle aussi, jouissait d'un talent pour le chant :

Il se fit une cabale pour introduire $\mathbf{M}^{\mathrm{lle}}$ de la Fayette à la place de $\mathbf{M}^{\mathrm{me}}$ de Hautefort [...] En peu de temps on vit que le roi ne parlait plus à Madame de Hautefort, et que

8Mercure galant, novembre 1679, 214-16 [NAF 11410, 53]. Les mots entre crochets suivis des initiales MB sont ceux de Marie Bobillier ; les autres sont les miens.

9 Mercure galant, mars 1680, 29 [NAF 11410, 55].

10 Mercure galant, mai 1688, 204-6 [NAF 11410, 112].

11 « Portrait de $\mathrm{M}^{\text {me }}$ de Hautefort sous le nom d'Olympe », Recueil des portraits et éloges en vers et en proses, dédié à S.A.R. Mademoiselle (Paris : Sercy et Barbier, 1659), 486 [NAF 11408, 726]. 
son grand divertissement chez la reine était d'entretenir $\mathrm{M}^{\text {lle }}$ de la Fayette et de la faire chanter ${ }^{12}$.

Même l'illustre $\mathbf{M}^{\text {me }}$ de Sévigné avoue vouloir s'adonner à la composition. Victor Cousin publie d'elle une lettre inédite adressée en 1656 à Ménage qui lui avait envoyé des vers italiens :

Vous sçavez que la petite Canzonnetta me parait la plus jolie du monde. Je tache de l'ajuster sur quelqu'un de tous les airs que j'ai jamais sçeus ; et n'y trouvant pas bien mes mesures, je pense que j'entreprendray d'y enfaire un tout neuf, tant $j$ ' ai d'envie de la chanter ${ }^{13}$.

Comme il était d'usage à l'époque, certains nobles maintenaient des musiciens et des musiciennes ; « Mlle de Guise [Marie de Lorraine] loge en son hôtel du Marais, à partir de 1680 environ [...], Marc Antoine Charpentier $»^{14}$ et quelques chanteuses. Le Mercure galant, d'ailleurs, en fait mention en parlant de M. de Montailly :

[...] un air noté : « Nos bois ont perdu leur feuillage », de M. de Montailly, maître à chanter qui apprend [à chanter] ${ }^{\mathrm{MB}}$ aux filles que $\mathrm{M}^{\text {elle }}$ de Guise entretient dans son hostel pour sa musique. Vous sçavez que cette magnifique princesse en a une fort bonne et qu'il y a presque tous les jours concert chez-elle ${ }^{15}$.

Une famille moins illustre mais tout aussi intéressée à la musique fut celle des Bombelles. Le Marquis de Bombelles, ministre sous Louis XVI, s'occupe des intérêts de sa sœur musicienne, Marie Jeanne Renée de Bombelles, Marquise de Travenet, ainsi que ceux de la célèbre Élizabeth de la Guerre. Marie aurait pratiqué la musique déjà à un bas âge, puisque vers 1750 , on verse un paiement de deux livres à un menuisier de Bitche pour « une boite pour la viole de $M^{\text {elle }}$ de Bombelles $»^{16}$. Le Marquis de Bombelles, le 31 janvier 1779, écrit de Ratisbonne :

Je voudrais fort que Madame Élizabeth [de la Guerre] eût déjà son clavecin ; mais le plus tôt qu'il puisse être rendu à Versailles, ainsi que celui de Madame de Canillac, [le mieux ;] c'est pour le 10 ou le 12 de mars ${ }^{17}$.

Dans une lettre du 23 août 1782, la Marquise de Travenet écrit à son frère, le Marquis de Bombelles, qu'elle va passer huit jours à Viarmes :

12 Victor Cousin, «Extrait des mémoires de La Porte », Madame de Hautefort (Paris, 1856), 24 [NAF $11408,726]$.

13 Cousin, La Société française au XVII siècle, d'après le grand Cyrus de Mlle de Scudéry, 4 éd., 2 vol. (Paris : Didier, 1873), 2:187 [NAF 11408, 731].

14Benoit, Versailles et les musiciens du roi, 41.

15 Mercure galant, février 1688, 98 [NAF 11410, 87].

16 "Papiers de la famille de Bombelles, 1740-60", Inventaire sommaire des archives départementales Seine-et-Oise, E 238 [NAF 11414, 198].

17 « Papiers de la famille de Bombelles, 1777-82 », Inventaire sommaire des archives départementales Seine-et-Oise, E 424 [NAF 11414, 198]. 
[...] nous faisons venir le jeune Goëtz, qui nous accompagnera sur le piano ; nous portons aussi nos harpes ; nous jouerons deux petites pièces de $\mathrm{M}^{\mathrm{me}}$ de Genlis ${ }^{18}$.

Le Mercure galant, toujours avide de communiquer les nouvelles extraordinaires, se plaît à faire valoir les talents de demoiselles de la noblesse qui $s$ 'adonnent déjà assez jeune à la musique :

J'ai à vous parler d'une merveille de nostre sexe. C'est de $\mathrm{M}^{\text {elle }} \mathrm{de} S$. Olon, fille de M. de S. Olon, qui a esté ambassadeur vers le roy de Maroc. Cette femme, $D^{\text {elle }}$, qui n'a que 12 ans, joue très bien du clavessin, et sçait la musique en perfection. Vous en jugerez par le menuet que je vous envoie, et qui est de sa composition ${ }^{19}$.

[Congrès de Nimèque ; l'ambassadrice Colbert en fait l'ornement avec sa fille] ${ }^{\mathrm{MB}}$ [...] âgée de sept ans et demy, qui possède déjà toutes les qualités du corps et de l'esprit $[\ldots]$ jouë admirablement bien de plusieurs instrumens, danse à charmer $[\ldots]^{20}$

La jeune princesse d'Osnabrugg [Osnabrück], fille de la duchesse de ce nom, [...] n'a que dix à onze ans, sçait déjà les langues, danse très bien, ettouche le clavecin avec une grâce merveilleuse ${ }^{21}$.

[Mariage de Melle Marianne Chevalier, âgée de 13 ans et demi avec Sr. René Le Tellier, conseiller à la cour des aides [...] $]^{\mathrm{MB}}[. .$.$] elle joue du clavessin, comme les$ plus habiles maistres, peint assez bien et ne s'entend pas mal a dessigner $[s i c]^{22}$.

Marolles, dans son discours sur la ville de Paris, ne manque pas d'énumérer les musiciennes les plus connues de son temps :

Mais, s'il faut aussi nommer des Dames qui ont aimé la musique des voix et des instrumens, qui n'a point ouï parler de la harpe et du luth de feue $\mathrm{M}^{\mathrm{e}}$ de Joyeuse, de $\mathbf{M}^{\mathrm{e}}$ de Fiennes, de feue $\mathbf{M}^{\text {elle }}$ Paulet, de $\mathbf{M}^{\text {elle }}$ Dupuy, de $\mathbf{M}^{\mathrm{e}}$ de Sassi, sa fille, de $\mathbf{M}^{\text {elles }}$ du Bouchet, de la Barre, et des Nots, et de la nonpareille $\mathrm{M}^{\text {elle }}$ ''Enclos, si elle ne se fût point brouillé à la Cour ? En voilà autant que l'ancienne Grèce avait autrefois compté de Muses, sans parler de $\mathrm{M}^{\text {elles }}$ Boni et Hilaire, et de $\mathrm{M}^{\mathrm{e}}$ de $\mathrm{S}$. Thomas ${ }^{23}$.

\section{Les musiciennes à la cour}

Au milieu du XVII e siècle, l'accès d'une femme à la musique de la cour constitue encore un événement exceptionnel ; l'inscription des musiciennes à la musique de la Chambre du roi, pas encore à la Chapelle, s'effectue avec réticence et lenteur ${ }^{24}$. Plus tard on tolérera que des femmes chantent à la

18 « Papiers de la famille de Bombelles, 1777-82 ", Inventaire sommaire des archives départementales Seine-et-Oise, 1777-1782. E 407 [NAF 11414, 198].

19 Mercure galant, avril 1670, 215 [NAF 11410, 116].

20 Mercure galant, décembre 1678, 160 [NAF 11410, 45].

21 Mercure galant, septembre 1679, 277 [NAF 11410, 51].

22 Mercure galant, janvier 1684, 256 [NAF 11410, 87].

23 « De l'excellence de la Ville de Paris (Onzième discours) ", Mémoires de Michel de Marolles, abbé de Villeloin, avec notes historiques et critiques, éd. par Dufour, 3 vol. (Amsterdam, 1755 ; Paris : Quantin, 1879), 3:208 [NAF 11410, 550-51].

24Benoit, Versailles et les musiciens du roi, 252. 
Chapelle, mais faudra-t-il encore que le roi l'ordonne. Le passage suivant de l'abbé Oroux est typique des circonstances et de l'attitude de l'époque :

On n'a pas encore oublié que Louis [XIV] entendant un jour la Messe dans la Chapelle avec les princes et les princesses ses enfants, dès qu'il se fût mis à genoux au milieu de cette auguste et nombreuse famille, La Lande fit chanter son beau Motet : Beati omnes qui timent Dominum. Le Roi qui avait devant lui les paroles du psaume fut vivement attendri ; mais il ne put retenir ses larmes lorsqu'on chanta le verset : « filii tui sicut novellae olivarum in circuitu mensae tuae ", et le reste du psaume, dont l'application, en effet, ne pouvait être plus heureux. [sic] Dans le grand nombre de faveurs qui devinrent la récompense des talents de cet habile homme [Delalande], une seule nous étonne. Il avait une femme [Anne Renée Rebel] et deux filles [Jeanne et Marie-Anne] dont la voix était très belle. Elles avaient été reçues pour chanter aux appartemens dans les ballets et concerts de la cour. Le roi, qui aimait à les entendre, permit qu'elles chantassent encore à la chapelle des récits qu'on composait exprès pour elles. Ce mauvais usage d'admettre des femmes dans la musique de la chapelle a continué jusque sous le règne de Louis $\mathrm{XV}$; il faut esperer qu'il ne sera pas établi. [L'auteur dit en note, d'après le Mercure de juillet 1722, qu'à cette date la $\mathrm{D}^{\mathrm{lle}} \mathrm{Hortense}$ des Jardins fut reçue dans la musique de la chapelle et de la chambre du roi, après avoir chanté à la messe, devant S.M. le verset Benedictus du psaume 123. $]^{\mathrm{MB} 25}$

Si, au début du XVIII ${ }^{e}$ siècle, un certain nombre de chanteuses entre au service du roi, surtout à la Chambre et quelques-unes à la Chapelle, on résiste toujours à l'admission de musiciennes instrumentistes, même celle d'une famille si illustre que celle des Couperin. Marguerite Louise, fille de François l'ancien, « une des plus célèbres musiciennes de nos jours, qui chantoit d'un goût admirable, et jouoit parfaitement du Clavecin [...] », selon Titon du Tillet, entre comme voix de dessus dans la musique de la Chambre du roi, en $1702^{26}$. Par ailleurs sa distante cousine, Marguerite Antoinette, fille de François Couperin le Grand, fut la première femme à occuper en 1736, la charge de claveciniste du roi et maître de clavecin des Enfants de France : [M $\mathrm{M}^{\text {lle }}$ Couperin] doit à son savoir et à la renommée de son illustre père [François], la place qu'elle occupe auj[ourd'hui] au concert de la Reine ; place d'autant plus flatteuse pour elle, que c'est une charge de la chambre que les femmes n'ont jamais exercée ${ }^{27}$.

Malheureusement, pour des raisons de santé, à ce qu'on dit, Marguerite Antoinette ne pourra pas enter en fonction, d'autant plus que cette charge sera transformée en une " commission ordinaire » la même année ${ }^{28}$. Selon un chargé d'affaires de la chambre du roi, des raisons pragmatiques et un attitude plus tolérante semblent prévaloir plus tard dans le siècle :

25 Histoire ecclésiastique de la cour de France, où l'on trouve tout ce qui concerne l'histoire de la chapelle, et des principaux Officiers ecclésiastiques de nos rois, par l'abbé Oroux, chaplain du roi, Abbé de Fontaine-le-Comte, 2 vol. (Paris, 1776-77), 1:522-23 [NAF 11408, 212-13].

26Benoit, Versailles et les musiciens du roi, 253-54, 263.

27Pierre-Louis d'Aquin de Chateau-Lyon, Lettres sur les hommes célèbres dans les sciences, la littérature, et les beaux-arts, sous le règne de Louis XIV (Amsterdam et Paris, 1752), 126 [NAF 11418, 322].

28 Voir Benoit, Versailles et les musiciens du roi, 199. 
Samedi, 13 novembre 1765. [...] Je me suis occupé d'un mémoire qui m'a été demandé par M. le duc d'Aumont, pour l'admission des femmes de la musique du roi au service de la chapelle, ce qui serait peut être plus décent et coûterait moins cher.

Dimanche, $1^{\text {er }}$ décembre 1765 . J'ai remis mon mémoire à M. le duc d'Aumont. Je ne crois pas que l'on puisse contredire les raisons que je donne pour l'admission de ce projet. Il ne s'agit que d'en bien persuader MM. les Évêques, ou, pour mieux dire, il suffit que le Roi l'ordonne ${ }^{29}$.

Qu'est-il advenu de ce mémoire ? On l'ignore.

\section{Les musiciennes dans les institutions}

Les institutions de femmes telles que les couvents, les monastères et les écoles demeuraient une source importante d'activité musicale pour les femmes de l'ancien régime. Sauval, commentant sur diverses églises de Paris et comment leur acoustique serait propice à la musique, s'arrête à l'église du Monastère des Filles de l'Assomption :

Ces religieuses ont des filles dans leur cloître qui chantent si agréablement, que la beauté et la douceur de leur voix attire tous les Samedis quantité de beaux monde à leurs litanies ${ }^{30}$.

En 1654, le Cardinal de Retz, se trouvant à Palma, capitale des îles de Baléares, raconte avoir entendu chanter dans un couvent de filles de la ville.

Le vice-roi me donna un magnifique dîner dans une superbe tente de brocard d'or qu'il avait fait élever sur le bord de la mer. Il me mena après entendre une musique dans un couvent de filles qui ne cédaient point en beauté aux dames de la ville. Elles chantèrent à la grille, en honneur deleur saint, des airs et des paroles plus galantes et plus passionnées que ne sont les chansons de Lambert ${ }^{31}$.

À l'École de Saint-Cyr, maison d'éducation pour jeunes filles fondée en 1686 par Louis XIV et $\mathbf{M}^{\text {me }}$ de Maintenon, l'importance de la musique dans la formation des étudiantes est soulignée par le Mercure galant dans un rapport sur Esther de Moreau :

Il y a des chœurs dans cette pièce de vingt quatre filles de St-Cyr, fait par M. Moreau, qui sont d'une grande beauté, et fort utiles à celles qui prennent le party de la religion, puisqu'elles apprennent par là à chanter, ce qui est très nécessaire dans les couvents ${ }^{32}$.

29 Journal de Papillon de la Ferté, intendant et comptrôleur de l'argenterie, menus plaisir et affaires de la Chambre du roi (1756-1780), publié avec une introduction et des notes par Ernest Boysse (Paris : Ollendorf, 1887), 177 [NAF 11413, 775].

30H. Sauval, Histoire et recherches des Antiquités de Paris, 2 vol. (Paris, 1724), 1:445 [NAF 11408, 234-35].

31 Euvres du Cardinal de Retz, tome 4, Édition des grands écrivains de France (Paris : Hachette, 1870), 557 [NAF 11410, 587].

32 Mercure galant, janvier 1689, 382 [NAF 11410, 116]. 
$M^{\text {me }}$ la Marquise de Sévigné est tout aussi impressionnée par ce qu'elle voit et entend à Saint-Cyr, où elle fait sa cour :

Je fis la mienne l'autre jour, à Saint-Cyr, plus agréablement que je n'eusse jamais pensé. [... $]^{\mathrm{MB}}$ Nous écoutames, le maréchal [de Bellefonds ${ }^{\mathrm{MB}}$ et moi, cette tragédie avec une attention qui fut remarquée, $[\ldots]^{\mathrm{MB}}$ Je ne puis vous dire l'excès de l'agrément de cette pièce. C'est une chose qui n'est pas aisée à représenter et qui ne sera jamais imitée ; c'est un rapport de la musique, des vers, des chants, des personnes, si parfait et si complet, qu'on n'y souhaite rien. Les filles qui font des rois et des personnages sont faites exprès. On est attentif, et on n'a point d'autre peine que celle de voir finir une si aimable piece ${ }^{33}$.

Au siècle suivant, on sollicite pour une abbaye les services professionnels d'une musicienne et on en fait l'annonce suivante :

$\mathrm{M}^{\mathrm{me}}$ l'Abbesse de Farmoutier desirerait avoir dans son Abbaye une Demoiselle qui sçût très bien toucher de l'Orgue, jouer du Clavecin, et qui fût en état d'enseigner la Musique et l'Accompagn[ement]t. Si cette Demoiselle veut être Religieuse, on la recevera dans la maison, sans dot ; sinon, on lui donnera des appointemens. Il faut s'adresser à Paris, à M. Balbastre, organiste de S. Roche, rue d'Argenteuil ${ }^{34}$.

\section{Les musiciennes professionnelles}

Il est certain que beaucoup de femmes pratiquaient la musique instrumentale, tant chez les aristocrates que chez les bourgeois, surtout au XVIII ${ }^{e}$ siècle. Mais en général très peu avaient accès à la profession, même si elles atteignaient un très haut niveau d'exécution. Rares étaient celles qui trouvaient emploi comme claveciniste ou organiste ; plusieurs enseignaient, et quelques-unes s'adonnaient à la composition ${ }^{35}$.

$\mathrm{M}^{\mathrm{r}}$ Molière [Mollier] a fait aussi une manière de petit opera qu'il donne en concert chez lui tous les jeudis depuis six semaines. Les assemblées y sont toujours plus illustres que nombreuses, le lieu estant trop petit pour contenir tous ceux qui viennent y demander place. Les vers en sont naturels, coulans, et propres à être chantez. Andromède attachée au rocher et délivrée par Persé, en est le sujet. Cette malheureuse princesse est représentée par Mademoiselle Itié [Marie Blanche Itier], fille de Molière[Mollier] qui chante avec toute la justesse possible. $\mathrm{M}^{\text {Ue }}$ Siglas [... ${ }^{\mathrm{MB}}$ fait le personnage de la mère $[. . .]^{\mathrm{MB}}$ La symphonie est agréablement diversifiée, selon les différentes passions qui se doivent exprimer. La merveille de notre siècle, la petite mademoiselle Jaguier [Élizabeth Jaguet de la Guerre] y touche le clavecin, et ce charmant divertissement finit par un air que chante une demoiselle de Normandie, qui a la voix admirable $[. . .]^{36}$

33 Lettres de Mme de Rabutin-Chantal, marquise de Sévigné à sa fille et à ses amis, édition revue et publiée par V. Silvestre de Sacy, 11 vol. (Paris, 1861), 9:205 [NAF 11410, 646].

34Annonces, affiches et avis divers. Feuille hebdomadaire, du 3 mai 1752 au 23 décembre 1761 (27 juin 1759) : 104 [NAF 11415, 381].

35Benoit, Versailles et les musiciens du roi, 254-60 ; Sadie, «Musiciennes of the Ancien Régime », 200-203.

36 Mercure galant, décembre 1678, 126-28 [NAF 11410, 44-45]. 
Il était peut-être plus facile pour une femme d'obtenir un poste d'organiste en dehors de Paris, comme le suggèrent les cas suivants :

En 1637 mourut le frère Jean de Saint-Lambert, carme déchaussé, aveugle dès le berceau, et qui remplissait les fonctions d'organiste ; en 1664, c'était une femme, Cécile Chaumette, qui tenait l'orgue de la cathédrale ${ }^{37}$.

En 1739, 102 l[ivres] et 11 sous à $\mathrm{M}^{\mathrm{me}}$ Badosse [ou Bodosse], organiste ${ }^{38}$.

D'Aquin trace un tableau des musiciennes qu'il juge avoir été les plus éminentes du règne de Louis XIV :

Plusieurs femmes dans le dernier siècle ont excellé dans le clavessin [sic]. $\mathrm{M}^{\text {le }} \mathrm{de}$ la Guerre a tenu un rang distingué parmi elles. Cette célèbre fille a donné un opéra et plusieurs cantates, qui prouvent la fertilité de son génie. M. Titon du Tillet l'a placée sur son Parnasse, parmi les musiciens les plus fameux ; il a fait exécuter son médaillon, et l'a embelli de cette légende :

Aux grands musiciens, j'ai disputé le prix.

$\mathrm{M}^{\mathrm{Mle}}$ Certin a fait aussi dans son temps, l'admiration de Paris ; elle était fort liée avec Lully, et ce grand homme lui faisait jouer sur le clavessin toutes les symphonies de ses opéras - vers du poète Lainés sur l'harmonie d'un clavecin d'André Ruckers :

Je suis fille du génie

Qui sous le beau nom d'harmonie

Réunis dans mes sons tous les charmes du chant

Et respectant les lois du Dieu qui m'a formée,

Je reste dans Rukers [sic] captive et renfermée

Et j'attends pour sortir la Certin ou Marchand.

$\mathrm{M}^{\mathrm{me}}$ de Plante et $\mathrm{M}^{\mathrm{ll}}$ Guyot, mortes en 1728 , touchaient de cet instrument avec toute la délicatesse possible ; elles étaient d'autant plus admirables, qu'elles savaient la composition et qu'elles préludaient avec la hardiesse et le succès des plus grands maîtres. $\mathbf{M}^{\mathrm{me}}$ du Hallay, décédée depuis plusieurs années, élève de Daquin, chantait les airs italiens avec le plus grand gout et la plus grande légèreté ; elle jouait du clavecin ; sa maison était le rendez-vous des artistes ; Largillière âgé de 82 ans a peint son portrait ; $M$. Desforges-Millard lui a adressé une ode [...] avec deux autres pièces de vers. $\mathbf{M}^{\text {lle }}$ Couperin doit à son savoir et à la renommé de son illustre père, la place qu'elle occupe aujourd'hui au concert de la reine ; [...] $\mathbf{M}^{\text {me }}$ Lévi et $\mathbf{M}^{\mathrm{me}}$ Haubault ont montré du talent sur le pardessus de viole, et ont joué avec succès au Concert spirituel ${ }^{39}$.

36Mercure galant, décembre 1678, 126-28 [NAF 11410, 44-45].

37La Champagne encore inconnue: documents curieux et inédits, publiés par Alexandre Assier, 2 vol. (Paris, 1876), 2:39 [NAF 11407, 629].

38 Inventaire sommaire des archives départementales d'Ardennes, antérieures à 1790, par MM. Sénemaud et Laurent, 4 vol., 4:G 103, 46 [NAF 11414, 13].

39D'Aquin de Chateau-Lyon, Lettres sur les hommes célèbres, 122-26 [NAF 11418, 321-22]. 
Le Concert spirituel, surtout dans la seconde moitié du XVIIIe siècle, fournira aux femmes musiciennes une occasion unique où les plus talentueuses d'entre elles pourront se manifester, aussi bien en tant que chanteuse qu'instrumentiste. Le Mercure rapporte un incident plutôt curieux au Concert spirituel du $1^{\text {er }}$ novembre 1783 ; le programme comprenait une nouvelle symphonie de Haydn, entre autres, et des petits airs variés sur la harpe par $\mathbf{M}^{\text {me }}$ Krumpholtz-Streckler qui, ce jour-là, joue :

non pour faire connaitre ses talents, dont elle a donné souvent de plus grandes preuves, mais pour détruire un préjugé répandu dans le public contre cet instrument. Plusieurs accoucheurs interdisent la harpe aux femmes enceintes. $\mathrm{M}^{\mathrm{me}} \mathrm{Kr}$ [umpholtzSteckler], convaincue qu'elle n'est nullement dangereuse dans cet état, a voulu en donner une preuve publique en en jouant elle-même, quoique au moment d'accoucher ${ }^{40}$.

En 1692, Du Pradel, dans son livre des adresses de Paris, ne mentionne que quelques femmes parmi plusieurs « Maîtres » pour la musique :

Maîtres pour le clavecin : [...] Mesdames Oves, rue St Denis, et Louis, rue de la Monnayé. Et encore Mesdemoiselles Rebour et Le Tellier, fauxbourg St Germain.

Maîtres pour la violle : [...] Mademoiselle Mengey, rue St Honoré, près la rue des Poullies, fait aussi profession de toucher et de montrer à toucher la violle ${ }^{41}$.

Par comparaison, une liste semblable qui date de 1756, démontre un nombre plus considérable de musiciennes professionnelles et de « Maistresses » :

Maistresses [de clavecin] : Madame Venière, [pas d'adresse] ; Mlle de Mars, rue St Thomas du Louvre ; Mlle Dubreuil, rue S. Sauveur ; Mme Bandeu, rue Honoré Chevalier ; Mad. Obtu, rue du Jardinet ; Mad. Dupré, cloitre S. Benoit ; Mad. Densez, rue du gros Chevet ; Mad. Laporte, rue des Prouvaires ; Mad. Dupont, au couvent de la Roquette ; Mad. Hérissé, rue du petit Lyon.

Tous [toutes] les organistes enseignent à jouer du clavecin.

[Maîtresse de] Guitarre [...] : Mlle Sonnet, rue Montmartre.

Maistresses de par dessus de viole : Mlle Lévy, rue de Prouvaires ; Mad. Dupont, [n.a.] ; Mad. La Roche, rue Fromenteau ${ }^{42}$.

40Mercure galant, novembre 1783, 131-32 [NAF 11410, 517].

41 Livre commode des adresses de Paris pour 1692 par Abraham du Pradel [Nicolas de Blégny] suivi d'appendices, précédé d'une introduction et annoté par Édouard Fournier, 2 vol. (Paris, 1878), 1:208-9 [NAF 11408, 851]. L'édition originale de 1691 et la deuxième édition de 1692 sont reproduites dans Benoit, Versailles et les musiciens du roi, 412-14.

42 Tableau de Paris pour l'année mil sept cent cinquante neuf, formé d'après les antiquités, l'histoire, la description de cette ville, etc... (Paris : Herissant, 1759), 206-9 [NAF 11414, 404-5]. 
Citons enfin, pour illustrer l'émancipation de la musicienne au temps de la Révolution, ce passage dans la presse du jour. Une jeune demoiselle de quinze ans, adresse courageusement la Convention nationale, en 1794 ; elle félicite les législateurs d'avoir créé l'Institut national de musique, et demande que soit fondé un lycée pour l'enseignement de la musique aux jeunes filles :

Ordonnez aussi, législateurs, que des places d'instituteurs et d'institutrices de ce lycée seront données au concours, et que les deux sexes pourront également y prétendre ; moi-même je ne craindrai pas de me mettre sur les rangs ; car pour une lutte aussi belle, si la carrière vient une fois à s'ouvrir, je veux être la première à m'y élancer. Alors, si je n'obtiens pas la palme de la victoire, j'aurai du moins l'avantage de l'avoir disputée à 15 ans ${ }^{43}$.

\section{Les prodiges}

Deux cas d'enfants prodiges, parmis d'autres, ont retenu l'attention des auteurs du XIX ${ }^{e}$ siècle : l'un au début et l'autre vers la fin de la période des Bourbon. Le premier date du temps d'Henri IV. L'enfant se nommait Angélique Paulet. Victor Cousin y attache une importance particulière, en citant de longs passages du Grand Cyrus de $\mathrm{M}^{\text {lle }}$ de Scudéry ${ }^{44}$, où elle apparaît sous le nom d'Élise. Selon Marie Bobillier, Cousin écrit qu'elle était née en 1591 ou 1592, fille de Charles Paulet, un des secrétaires d'Henri IV, et l'inventeur de l'impôt appelé : « Paulet ». Puis Cousin offre un portrait de la jeune enfant :

Sa beauté la fit remarquer de très bonne heure et, toute jeune encore, elle eut les plus grands succès à la cour galante d'Henri IV. Son père, qui avait de l'ambition, releva encore les agréments de sa fille en lui donnant toutessortes de maîtres qui développèrent ses moyens de plaire. Elle dansait et chantait à ravir, et touchait du luth avec un rare talent [...] Voici le portrait qu'en fait Tallemant, très peu porté, comme on sait, à l'admiration : « $\mathbf{M}^{\text {Ile }}$ Paulet avait beaucoup de vivacité, était jolie, avait le teint admirable, la taille fine, dansait bien, jouait du luth et chantait mieux que personne de son temps. On raconte que l'on trouva deux rossignols morts sur le bord d'une fontaine où elle avait chanté tout le jour ; mais elle avait les cheveux si dorés qu'ils pouvaient passer pour roux. $»^{45}$

Toujours selon Cousin, $\mathbf{M}^{\text {lle }}$ Paulet dut paraître pour la première fois vers $1625[?]^{\mathrm{MB}}$ ou 1626 à l'hôtel de Rambouillet. Sa belle voix et son talent de musicienne lui donnaient un rang à part dans les divertissements qui s'y donnaient. Cousin cite l'extrait des mémoires d'Arnault, relatif à la représentation de Sophoniste de Mairet, où $\mathbf{M}^{\text {lle }}$ Paulet paraissait dans les entractes en chantant et en s'accompagnant sur le théorbe.

43 « Petition faite à la Convention nationale par la $\mathrm{Cit}^{\text {ne }}$ Heuvrard, dans la séance du 21 nivrose ", Gazette nationale ou le Moniteur universel, $\mathrm{n}^{\circ} 128$ (oclidi 8 pluviose, an II [lundi 21 janvier 1794]) : 310-11 [NAF 11415, 323-24].

44Madeleine de Scudéry, Artamène ou le Grand Cyrus (Paris, 1649-63), 4:229-30 ; 7:216-18.

45Cousin, La société française au XVII siècle, 283-84 [NAF 11408, 727-28]. 
$\mathrm{M}^{\text {elle }}$ Paulet, habillé [sic] en nymphe, chantait avec son théorbe entre les actes, et cette voix admirable, dont on a assez ouï parler sous le nom d'Angélique, ne nous faisait point regretter la meilleure bande de violons qu'on emploie d'ordinaire en ces intermèdes ${ }^{46}$.

Dans le Grand Cyrus, $\mathbf{M}^{\text {lle }}$ de Scudéry décrit la cour de France du temps d'Henri IV sous le nom de « royaume de Phénice » et Cousin en extrait ce qui est relatif à Mlle Paulet :

Son père, appelé Straton, était extrêmement riche [...] C'était chez lui qui se faisaient toutes les parties de plaisir, soit de promenade, de musique ou des festins [...] Elle était née avec une si belle voix et une telle disposition à la danse que dès l'âge de cinq ans elle chantait juste et dansait en cadence, commençant même de toucher la lyre, mais avec tant de grâce qu'elle charmait tous ceux qui la voyaient. [...] Un Tyrien appelé Crysile (Pierre Guédron) qui savait la musique admirablement, et qui était allé voyager, revint à Tyr (Paris); et comme c'était un fort honnête homme et comme toute la cour, il fut chez Straton (Paulet) comme chez les autres ; il fut si charmé de la jeune Élise (Angélique Paulet) qu'il voulut être son maître et lui enseigner pour la lyre et pour tout ce qu'il avait appris d'Arion avec qui il avait amitié particulière ${ }^{47}$.

S'appuyant sur ce que raconte Malherbe, Marie Bobillier suppose que $\mathbf{M}^{\text {lle }}$ Paulet aurait connu son premier grand succès à la cour dans un bal donné en 1609 , où elle parut montée sur un Dauphin, jouant et chantant le rôle d'Arion. Ceci correspond à la longue description de cette " fête " dans le Grand Cyrus de $\mathrm{M}^{\text {lle }}$ de Scudéry, que cite Cousin. Cependant, au lieu de citer intégralement Cousin, Bobillier donne un résumé du contexte de ce bal avant de citer l'auteur : «La Fête en question fut donnée en l'honneur du Prince de Condé qu'Henri IV aimait. Les aventures d'Arion furent choisies probablement en raison des machines et des décors de la scène [...] Guédron propose Mlle Paulet pour le rôle d'Arion ; le Roi obtient le consentement de son père et Guédron lui enseigne son rôle et est étonné de la facilité avec laquelle elle l'apprend ». Bobillier cite ensuite Cousin :

Lorsque la jeune Élise parut sur le Dauphin qui la portait, toute l'assemblée fit un cri d'admiration [...] Elle tenait sa lyre avec une grâce admirable, et chantait avec une assurance et une justesse si merveilleuse que toute la cour en était surprise et charmée. [...] en effet, c'était une chose étonnante de voir que la voix d'une si jeune personne pût avoir assez d'étendue pour remplir un aussi grand lieu que celui-là, et pour le remplir d'une harmonie si charmante et si capable de toucher les cœurs $[. . .]^{48}$

C'est à la fin du XVIII' siècle que l'on retrouve la seconde enfant prodige. En 1813, le Général Thiébault loue l'habilité des femmes à composer des romances et cite en exemple la jeune $\mathbf{M}^{\text {lle }}$ de Saint-Ser : 
Mais les dames auxquelles le succès des romances est confié aujourd'hui, n'ont pas voulu se borner à la gloire de les faire valoir, elles ont voulu partager celle d'en faire ; et leur sensibilité vive, leur organisation fine et délicate, leur imagination en général active et souvent ardente, leur ont rendu à cet égard l'emploi du talent facile, ainsi qu'elles l'ont prouvé par un grand nombre de romances nées des plus heureuses inspirations. Nous citerons à l'appui de ce fait $\mathbf{M}^{\text {Ile }}$ Odile de Saint-Ser, génie aussi heureux que précoce en musique, et qui, en 1788 alors âgée de 13 ans, fit une romance pleine de grâce, d'expression et de talent, sur ces paroles[ :] « Est-ce pour moi que tu verses des larmes, sensible aurore », etc. [...] L'auteur fait la note suivante :

(1) Cette $\mathrm{M}^{\text {elle }} \mathrm{de}$ St Ser était regardée comme un prodige ; dès qu'elle put mettre les mains sur le clavier, elle toucha du piano et composa ; elle avait fait à 8 ans un air de tambourin qu'on regardait comme un chef d'œuvre ; à 12 ans elle avait plusieurs œuvres de musique gravées chez Parso. [Il explique comment Mme de Saint-Ser, sa mère, demanda à Sacchini d'enseigner à sa fille mais qu'il avait répondu qu'il ne connaissait personne qui pouvait lui enseigner et qu'il était mieux de la laisser à elle-même. ${ }^{\mathrm{MB}}$. Mme de Saint-Ser recevait tous les Vendredi : beaucoup de monde se réunissait chez elle, et notamment quelques personnes de marque ; tous s'extasiaient sur le compte de cette jeune personne, d'autant plus étonnante qu'elle ne jouait que de la musique de sa composition ; mais l'enthousiasme qu'elle excitait ne produisait sur elle aucun effet ; et sans paraitre se douter qu'elle fut l'objet de tant d'applaudissement, elle laissait le piano pour reprendre sa poupée. En 1789, Mme de St Ser quitta Paris avec sa fille et depuis je n'en ai plus eu que des nouvelles vagues et très incertaines ${ }^{49}$.

Thiébault fait mention plus loin d'une demoiselle Aurélie Gardel qui « fit, très jeune aussi, cette jolie romance : " De ce ruisseau l'onde parait plus pure " " ${ }^{50}$, tandis que le Mercure galant rapporte qu' une "Mlle Carlin, aveugle, âgée de 13 ans, fille de l'acteur Carlin, élève de Romain, joua un concerto de Bach [Emmanuel ?] ${ }^{\mathrm{MB}}$ au Concert Spirituel du 1 novembre $1778^{51}$.»

Ces commentaires sur l'activité musicale des femmes de l'ancien régime par divers auteurs des trois derniers siècles et rassemblée un peu au hasard par Marie Bobillier constitue un document assez exceptionnel que l'on serait porté à associer aux sondages d'opinions modernes. En effet, à notre avis, l'analogie ne semble pas trop poussée, et il serait tout à fait légitime de vouloir en tirer quelques lumières conclusives. Hélas, comme le lecteur l'aura déjà constaté, le sondage ne sert qu'à confirmer ce que d'autres ont conclu sur le sort de la femme musicienne des XVII et ${ }^{\mathrm{XVIII}}$ e siècles. Pas plus dans le domaine de la musique que dans d'autres activités artistiques, politiques ou sociales se trouve-t-elle privilégiée par rapport à sa contrepartie masculine. En grande part, la profession en musique appartient à la gent masculine ; et s'il y a quelques exceptions, plus dans le domaine de l'exécution que celui de la composition, et plus au XVIII siècle qu'au XVII', ce n'est que trop souvent grâce à un talent exceptionnel joint à un lien de parenté célèbre. Il faudra attendre la seconde moitié du $\mathrm{XX}^{\mathrm{e}}$ siècle

$49 \mathrm{Du}$ chant et particulièrement de la romance, par le général Thiébault, baron de l'Empire (Paris : Bertrand, 1813), 106 [NAF 11418, 336-37].

50 Ibid., 107 [NAF 11418, 338].

51 Mercure galant, novembre 1778 [NAF 11419, 52]. 
pour que la femme musicienne puisse enfin commencer à trouver sa juste place à part égale avec l'homme musicien dans les divers rangs de la profession.

\section{Résumé}

À sa mort en 1918, Marie Bobillier (pseud. Michel Brenet) a laissé au Département des manuscrits de la Bibliothèque nationale à Paris un nombre impressionnant de notes, de citations et de relevés qu'elle avait accumulés au cours de sa carrière et qui, pour la plupart, demeurent inédits. Dans un dépouillement de ces volumes, l'auteur a extrait les citations, les rapports et les commentaires des auteurs des trois derniers siècles, tous sous le thème «femmes musiciennes». Il en résulte un éventail assez étonnant des activités musicales des femmes de l'ancien régime sous les rois Bourbon, une espèce de sondage du passé à partir duquel tirer quelques conclusions plus éclairées. Malheureusement, le travail n'a servi qu'à confirmer ce que d'autres ont déjà conclu, à savoir que les femmes des XVII et XVIII' siècles n'avaient qu'un rôle secondaire en musique, tout comme dans les autres aspects de la société, soit artistique, politique ou social. Il faudra attendre le $\mathrm{XX}^{\mathrm{e}}$ siècle pour que les femmes puissent commencer à prendre leur juste place dans les rangs de la profession musicale. 